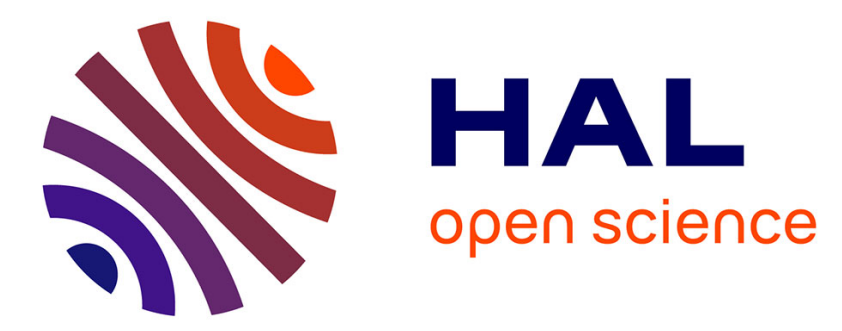

\title{
Low Power Radiolocation through Long Range Wide Area Networks: a performance study
}

Nicola Accettura, Samir Medjiah, Balakrishna Prabhu, Thierry Monteil

\section{To cite this version:}

Nicola Accettura, Samir Medjiah, Balakrishna Prabhu, Thierry Monteil. Low Power Radiolocation through Long Range Wide Area Networks: a performance study. 2017 IEEE 13th International Conference on Wireless and Mobile Computing, Networking and Communications (WiMob), Oct 2017, Rome, Italy. pp.1-8, 10.1109/WiMOB.2017.8115799 . hal-02063812

\section{HAL Id: hal-02063812 \\ https://hal.laas.fr/hal-02063812}

Submitted on 11 Mar 2019

HAL is a multi-disciplinary open access archive for the deposit and dissemination of scientific research documents, whether they are published or not. The documents may come from teaching and research institutions in France or abroad, or from public or private research centers.
L'archive ouverte pluridisciplinaire HAL, est destinée au dépôt et à la diffusion de documents scientifiques de niveau recherche, publiés ou non, émanant des établissements d'enseignement et de recherche français ou étrangers, des laboratoires publics ou privés. 


\title{
Low Power Radiolocation through Long Range Wide Area Networks: a performance study
}

\author{
Nicola Accettura, Samir Medjiah, Balakrishna Prabhu and Thierry Monteil \\ LAAS-CNRS, Université de Toulouse, CNRS, UPS, INSA, Toulouse, France \\ Email: \{accettura,medjiah,prabhu,monteil $\} @ l a a s . f r$
}

\begin{abstract}
Low Power Wide Area Networks (LPWAN) technologies have recently triggered many research efforts and standardization activities due to the inherent possibility of both providing long range wireless communications and guaranteeing a long life for very cheap sensing devices. At the same time, the increasing interest of telco operators into such kind of networks is due to the wide range of applications that can be supported by LPWAN, including low power radiolocation. Among the available LPWAN access schemes, LoRaWAN protocols enable a hierarchical network structure over sub-gigahertz unlicensed spectra, with coordinated gateways listening for data delivered by battery-operated end-devices. A proper positioning scheme for gateways enables radiolocation duties through multilateration. However, the LoRaWAN Aloha-based capacity puts an implicit restriction on the radiolocation performances, while explicit duty cycle policies further limit the resource availability on unlicensed frequencies. To characterize the throughput of low power radiolocation applications, this contribution introduces a scalable and detailed probability model, while validating the same through a large simulation campaign. The results clearly show that the availability of multiple channel enhances the capacity of LoRaWAN networks while insuring predictable time delay between consecutive successful radiolocation events.
\end{abstract}

\section{INTRODUCTION}

The progress in miniaturization techniques for electronic circuits is still advancing with the effect of an upcoming tidal increase both in the number of networked objects and in the amount of generated information traffic [1]. Wireless sensor networks have been the first embodiment of such technological development, with short range and lossy wireless links policed over the physical layer defined by the IEEE802.15.4 standard [2]. Reliability and time-criticity were used to constrain the design of communication architectures, with a wide fallout in research, development and standardization of synchronized low power mesh networks [3].

However, some low power applications would require very sporadic communications without constrains on bounded communication delays. This relaxation permits the use of low power long range communications, thus removing the need for relaying capabilities. As further consequence, processing units can be safely resized, thus allowing very cheap circuitry to be used for such applications. All in all, this led to the definition of Low Power Wide Area Networks (LPWAN) [4], with standardization efforts recently started for handling interoperable solutions [5].

Based on cellular-type architectures, LPWANs aim to provide long range connectivity on the top of unlicensed spectra, thus easing their deployment at a global scope [6]. Among the existing LPWAN technologies, LoRaWAN [7] can target specific application needs (simple reporting, reporting with simple feedback or complete bidirectional connectivity), thus resulting as the most flexible in terms of offered communication modes. LoRaWAN also achieves very long communication ranges by using Chirp Spread Spectrum techniques [8], [9]. Nonetheless, to achieve a fair use of the unlicensed spectrum among concurrent operators and/or LPWAN technologies, the radio access is policed through duty cycle limitation to be hard-coded on end-devices [10]. Furthermore, the LoRaWAN bandwidth is inherently limitated by the Aloha access scheme [11].

As matter of fact, LoRaWAN is a novel technology, that has triggered research studies about network performance. In [12], authors have made a first attempt to assess the performances of LoRaWAN focusing on network scalability aspects. Authors in [13], propose to study the uplink throughput of a single end-device. Moreover, they have studied the scalability aspect through a set of applications in order to derive the maximum possible number of end devices in the network. In [14], authors propose to study the capacity of a LoRaWAN network through the point of view of communication requirements. Remarkably, the throughput of LoRaWAN has not been studied to target scalable multi-channel multi-gateway deployments allowing low power radiolocation applications. In such environment, the classical Aloha probability models do not fit the network performance evaluation.

This paper presents a model description for the throughput in multi-channel multi-gateway Aloha networks allowing radiolocation. Such a model is also validated by results collected through extensive simulation campaigns. To this end, the event-based simulator LoRaWAN-sim has been developed in Python language jointly with the implementation of realistic traffic generation patterns complying with duty cycle policies dictated by the European Telecommunications Standards Institute (ETSI).

In what follows, Sec. II gives an overview on LoRaWAN, while Sec. III details the proposed throughput model for radiolocation purposes. Then, Sec. IV presents simulation results validating such a model. Finally, Sec. V concludes the paper and envisages future works.

\section{LORAWAN NETWORK ARCHITECTURE}

LoRaWAN defines the medium access scheme running on the top of LoRa hardware technology [8]. 
Architecturally, in a LoRaWAN network a central server coordinates several gateways. Each gateway relays traffic between low power end-devices and the server itself. Wireless communications between gateways and end-devices happen over unlicensed frequencies. Even though the LoRaWAN specification refers to such a network topology as a "star-ofstars" [7], a packet sent on the radio by any end-device can be received by more than one gateway: many copies of the same packet will be then delivered to the server, that in turn will discard replicas. To better represent such a topology, it is worth exploiting graph theoretical arguments. The union of all end-devices and all gateways is the set of nodes of the graph. If an end-device falls into the coverage range of a gateway, a link between the end-device and the gateway represents their possibility to communicate to each other. Thus, the set of edges of the graph is composed by all these links. In fact, it is very easy to figure out that the graph representing the wireless portion of the network is a bipartite one, with any edge connecting an end-device on a side and a gateway on the other one.

LoRaWAN simultaneously handles communications over many physical frequency channels. For each channel, several data rates are also available: the lower the data rate, the longer is the time-on-air of any sent packet, the higher is the insured decoding capability, the larger is the resulting transmission range [9]. However, data rates that are lower than the highest are not used in this contribution, because they do not target large LoRaWAN deployments. A justification for this choice is provided by the following example. The circular crown centered at the gateway and associated with the transmission range permitted by a low data rate is more extended than the circle centered at the gateway and associated with the transmission range permitted by the highest data rate [13]. If the density of end-devices per area is constant and all enddevices inject the same amount of traffic on the network, it follows that: (i) the number of end-devices transmitting at a low data rate is higher than the number of end-devices transmitting at the highest data rate; (ii) a packet is transmitted at low data rate in a longer time-on-air than the one resulting when using the highest data rate. As a consequence, the probability of collision when using a data rate lower than the highest is always increased ${ }^{1}$.

Conceptually, LoRaWAN moves complexity on the gateways and to the network server. Gateways decode packets received simultaneously on several channels. The network server is then responsible for discarding packet replicas received by many gateways. A packet correctly received by one or more gateways can be acknowledged by only one gateway selected by the network server on channels or sub-bands dedicated to downward traffic, thus featuring the LoRaWAN wireless communication as full-duplex.

In details, LoRaWAN permits three modes of operations. In Mode A, a bidirectional communication is started by any

\footnotetext{
${ }^{1}$ An interesting work confirming such a reasoning through simulations is available on line at https://github.com/maartenweyn/lpwansimulation/
}

end-device whenever it has a packet available in its output buffer, unless some hard-coded policy delays such a transmission in order to perform a duty cycle limitation [10]. The packet is transmitted on any frequency channel available for upward traffic. If a communication feedback is expected, the end-device will turn on its radio for two very short time windows listening to incoming acknowledgement packets. Data addressed to the end-device can be piggybacked to the acknowledgments. The rationale of Mode $A$ is a Pure Aloha access scheme addressing monitoring applications with very low traffic requirements, since a higher traffic would trigger many packet collisions and a poor performance. Instead, in Mode B, end-devices listen for beacons broadcast by gateways, so that they can schedule in advance time windows used by gateways to relay data to end-devices, thus allowing bidirectional communication started by the back-end. Indeed, Mode $B$ calls for resource scheduling to avoid packet collisions and waste of bandwidth. In addition, some time-critical applications could also be allowed, such as closed-loop networked control systems. Finally, in Mode $C$, when an end-device has got nothing to transmit, it will set its radio to listening mode.

In the remaining part of this contribution, it has been assumed that mode $\mathrm{A}$ is used for radiolocation purposes. Acknowledgements are not taken into account, since communications happening on a separate channel do not affect the traffic related to radiolocation. Finally, the resulting throughput probability model accounts for multiple channels, as per regional requirements [15].

\section{THROUGHPUT MODEL}

As described in the previous sections, the LoRaWAN protocol is based on a Pure Aloha medium access scheme. The performance of Aloha networks has been very well investigated in the past fifty years [11]. Nonetheless, the LoRaWAN framework holds some peculiar architectural features, whose joint performance has never been investigated from a detailed probabilistic point of view. Indeed, LoRaWAN enhances the Aloha-scheme by allowing the simultaneous exploitation of several channels at the same time. In addition, a packet transmitted by an end-device can be received by several gateways. Since one of the most desired applications expected by LoRaWAN operators is radiolocation of end-devices through signal multilateration, it is worth to investigate the expected throughput in such scenarios for low power radiolocation applications.

A very interesting work has investigated the throughput of Slotted Aloha networks with coordinated gateways [16]. The authors propose a model of the throughput based on the assumption that gateways are randomly spread on a given area according to a homogeneous Poisson point process and that end-devices can fall outside the coverage range of gateways. The throughput formulation is not straightforward and can be evaluated through approximations and numerical methods.

Contrariwise, in this paper it has been assumed that the gateways are spread non-randomly according to a specific tessellation principle: any end-device must be able to send a 
packet to at least three gateways. This assumption comes from the common sense observation that realistic cellular networks deployments aim to get a complete coverage of an area. To achieve generality and scalability, end-devices are still spread on a surface according to a Poisson point process. Due to the tessellation scheme, it is possible to find a unit area that repeats over an indefinitely wide surface: the throughput computed on that unit area divided by the unit area itself is in fact equal to an average normalized throughput of the considered system. For scalability purposes, the throughput over a given area is obtained as the product of such an area and the average normalized throughput.

The remaining part of this section describes the probability model for the throughput analysis of LoRaWAN networks, by first introducing the concept of density-based throughput.

\section{A. Density-based throughput}

It is reasonable to assume that the time between two successive packet generations is distributed as a memory-less random variable. For discrete-time systems, this assumption is easy to implement on real hardware by means of a geometric distribution. In order to allow for a more general setting of continuous-time systems, we shall assume that the interarrival times follow an exponential distribution, which is continuoustime counterpart of the geometric distribution. The average generation rate of packets is assumed to be equal to $\lambda$ packets per seconds. Defining $\tau$ as the time-on-air of a LoRaWAN packet (expressed in seconds) sent by a given end-device to the closest gateways, the probability $p$ that an end-device starts transmitting a packet on the air in a time interval shorter than $\tau$ seconds is:

$$
p=1-e^{-\tau \lambda}
$$

In a Pure Aloha access scheme, the probability that an enddevice starts transmitting a packet in a time interval shorter than $2 \tau$ [17] is equal to $1-e^{-2 \tau \lambda}$. Given that end-devices can randomly transmit in a pool of $n$ channels, the probability $q$ that a given end-device is not interfering with an ongoing transmission on a given channel (either it does not transmit, or it transmits on another channel) can be computed as:

$$
\begin{aligned}
q & =\operatorname{Pr}(\text { no tx })+\operatorname{Pr}(\text { tx on another channel }) \\
& =e^{-2 \tau \lambda}+\left(1-e^{-2 \tau \lambda}\right) \frac{n-1}{n} \\
& =1-\frac{1-e^{-2 \tau \lambda}}{n}=1-\frac{1-(1-p)^{2}}{n} .
\end{aligned}
$$

It has been assumed throughout this paper that end-devices are spread on an area of infinite extension on the basis of a homogeneous Poisson point process. Furthermore, it has been assumed that the unit area is a square with side equal to the maximum transmission range $R$, thus allowing for scalable deployments (i.e., $R$ is a parameter that depends only on the transmission technique inherent to the LoRa technology). As a consequence, any area $\mathcal{A}$ is expressed in multiples of such a unit area, and the variable $\mu$ indicates the average number of end-devices per unit area as well. It follows that the probability that $i$ end-devices are present on an area $\mathcal{A}$ is exactly:

$$
\operatorname{Pr}(i)=e^{-\mu \mathcal{A}} \frac{(\mu \mathcal{A})^{i}}{i !} .
$$

From these premises, the overall probability $Q(\mathcal{A})$, that none of the end-devices in a given area $\mathcal{A}$ is transmitting on a given channel, is obtained by using (3) according to the law of total probability as follows:

$$
Q(\mathcal{A})=\sum_{i=0}^{\infty} q^{i} \operatorname{Pr}(i)=e^{-\frac{(2-p) p \mu \mathcal{A}}{n}}
$$

while the overall throughput $S(\mathcal{A})$ related to a given area $\mathcal{A}$ is obtained as follows:

$$
S(\mathcal{A})=n \sum_{i=1}^{\infty} i \frac{p}{n} q^{i-1} \operatorname{Pr}(i)=p \mu \mathcal{A} \cdot Q(\mathcal{A}) .
$$

By substituting the area value with $\pi$ (i.e., the corresponding circle area around a single gateway) and by using a single channel in the previous formula, the throughput expression becomes $^{2}$ :

$$
S(\pi)=p \mu \pi \cdot e^{-(2-p) p \mu \pi} .
$$

Eventually, it is worth defining the throughput $S^{*}\left(\mathcal{A}_{x}, \mathcal{A}_{y}\right)$ related to an area $\mathcal{A}_{x}$, that accounts for the possible interference due to end-devices present into a larger area $\mathcal{A}_{y} \supset \mathcal{A}_{x}$ :

$$
S^{*}\left(\mathcal{A}_{x}, \mathcal{A}_{y}\right)=S\left(\mathcal{A}_{x}\right) \cdot Q\left(\mathcal{A}_{y}-\mathcal{A}_{x}\right)=p \mu \mathcal{A}_{x} \cdot Q\left(\mathcal{A}_{y}\right) .
$$

It comes easy to find that $S^{*}(\mathcal{A}, \mathcal{A})=S(\mathcal{A})$.

\section{B. Radiolocation model}

It is very common to model the arrangement of base stations according to a two-dimensional lattice. In this paper an hexagonal lattice is considered, as pictured in Fig. 1: three nearby points in the lattice form an equilateral triangle, e.g., the dashed equilateral triangle $A D E$, that in turn represents the unit area used for a regular triangular tessellation. In the figure, LoRaWAN gateways are placed at the point locations belonging to the lattice, and are indicated with black dots.

The transmission range of any gateway is indicated with $R$ and the resulting coverage area is a circle with radius $R$ (e.g., the area bounded by the black circumference centered on $A$ in Fig. 1). By assuming that $R$ is equal to the distance between two nearby gateways, it comes out that an end-device placed at any point on the plane falls into the coverage range of at least 3 gateways, thus allowing radiolocation on the whole domain of a LoRaWAN deployment. For the sake of clarity, the coverage area of each gateway in the figure is bounded by a gray dotted circumference centered on the gateway itself. Two sub-areas important for the arguments used hereafter are also highlighted:

1) the end devices in the dark grey shape, whose related area is $\mathcal{A}_{t}=(\sqrt{3}-\pi / 2)$, will be able to communicate

\footnotetext{
${ }^{2}$ The product $p \mu \pi$ is exactly the traffic $G$ offered to the network, so that, by letting $G$ staying constant in the limit $p \rightarrow 0$ and $\mu \rightarrow \infty$, the throughput value settles to $G \cdot e^{-2 G}$, as in the classic Pure Aloha formulation [11].
} 


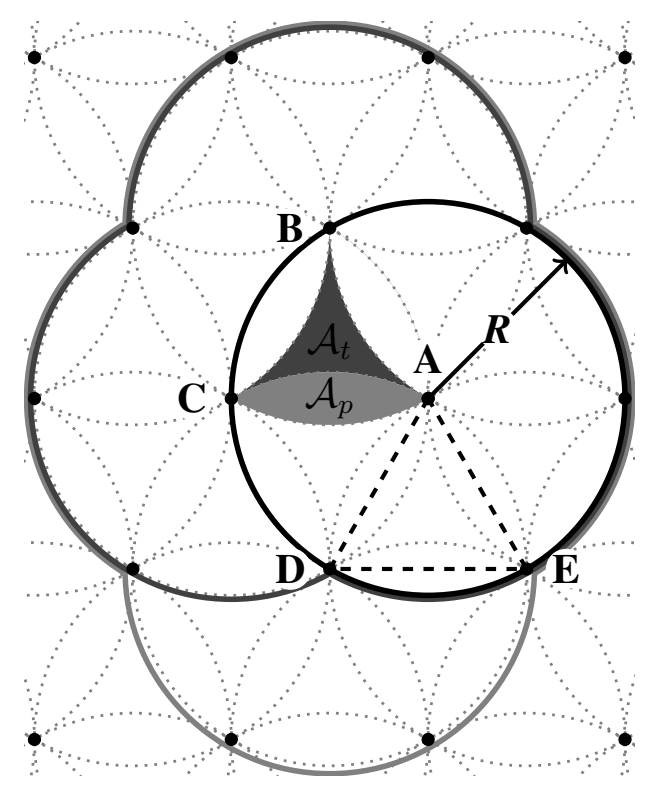

Fig. 1. Model for the displacement of LoRaWAN gateways and radio coverage.

with 3 gateways; in the considered example, those gateways are placed in $A, B$ and $C$;

2) the end devices in the light grey shape, whose related area is $\mathcal{A}_{p}=(\pi / 3-\sqrt{3} / 2)$, will be able to communicate with 4 gateways; in the considered example, those gateways are placed in $A, B, C$ and $D$.

As it can be inferred by graphical inspection on Fig. 1, a proper combination of these sub-areas can periodically tessellate the plane in an another equivalent way.

Remarkably, the unit area of the regular triangular tessellation, e.g., the equilateral triangle $A D E$, is equal to $\left(\mathcal{A}_{t}+1.5 \mathcal{A}_{p}\right)$. Defining $\Gamma_{t}^{r}$ and $\Gamma_{p}^{r}$ as the throughput values for radiolocation calculated respectively on $\mathcal{A}_{t}$ and $\mathcal{A}_{p}$, the average normalized throughput of the LoRaWAN deployment is given by the overall success rate $\left(\Gamma_{t}^{r}+1.5 \Gamma_{p}^{r}\right)$, related to devices in that unit area, divided by the triangular area itself. For the sake of comparison with the single-gateway case of (6), the average throughput expression has been given for a circular area (extended as $\pi$ units of areas) picked anywhere in the LoRaWAN deployment. Such a throughput, $\Gamma^{r}$, is then formulated as follows:

$$
\Gamma^{r}=\frac{4 \pi}{\sqrt{3}}\left(\Gamma_{t}^{r}+1.5 \Gamma_{p}^{r}\right)
$$

To derive $\Gamma_{t}^{r}$ and $\Gamma_{p}^{r}$, it is worth introducing some definitions used hereafter (by referring to Fig. 1):

- $\mathcal{A}_{3}=(3 \pi / 2+\sqrt{3})$ is the area covered by 3 gateways placed at the vertices of an equilateral triangle with side equal to $R$ (e.g., the union of the circles centered at $A$, $B$ and $C)$;

- $\mathcal{A}_{3+}=(5 \pi / 3+\sqrt{3})$ is the area covered by 3 gateways with two of them being (i) distant from each other by more than $R$ but less than $2 R$, and (ii) having a distance

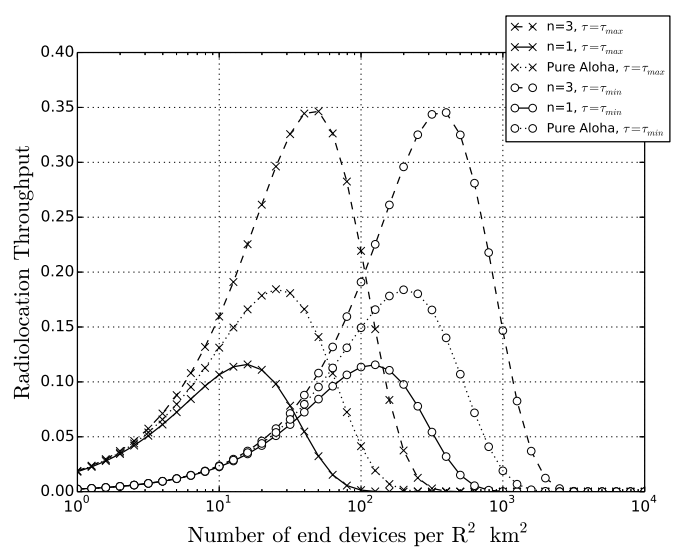

Fig. 2. Throughput comparison of LoRaWAN network with classic Pure Aloha ones.

equal to $R$ from the third (e.g., the union of the circles centered at $A, B$ and $D)$;

- $\mathcal{A}_{4}=(5 \pi / 3+3 \sqrt{3} / 2)$ is the area covered by 4 gateways placed at the vertices of a $60^{\circ}$ rhombus with side equal to $R$ (e.g., the union of the circles centered at $A, B, C$ and $D$ ).

$\Gamma_{t}^{r}$ is computed as the throughput $S^{*}\left(\mathcal{A}_{t}, \mathcal{A}_{3}\right)$ related to the area $\mathcal{A}_{t}$ and subject to the interference of the end-devices in the total area covered by the 3 gateways:

$$
\Gamma_{t}^{r}=p \mu \mathcal{A}_{t} \cdot Q\left(\mathcal{A}_{3}\right)
$$

On the other hand, $\Gamma_{p}^{r}$ is computed according to the inclusion-exclusion principle as follows:

$$
\Gamma_{p}^{r}=p \mu \mathcal{A}_{p} \cdot\left[2 Q\left(\mathcal{A}_{3}\right)+2 Q\left(\mathcal{A}_{3+}\right)-3 Q\left(\mathcal{A}_{4}\right)\right]
$$

Combining (8) with eqs. (4), (9) and (10), the resulting throughput expression is given by:

$$
\begin{aligned}
\Gamma^{r}=p \mu \pi \cdot[ & \left(\frac{2 \pi}{\sqrt{3}}-2\right) e^{-\frac{(2-p) p \mu \pi}{n}\left(\frac{3}{2}+\frac{\sqrt{3}}{\pi}\right)}+ \\
& +\left(\frac{4 \pi}{\sqrt{3}}-6\right) e^{-\frac{(2-p) p \mu \pi}{n}\left(\frac{5}{3}+\frac{\sqrt{3}}{\pi}\right)}+ \\
& \left.+\left(-\frac{6 \pi}{\sqrt{3}}+9\right) e^{-\frac{(2-p) p \mu \pi}{n}\left(\frac{5}{3}+\frac{3 \sqrt{3}}{2 \pi}\right)}\right]
\end{aligned}
$$

To shed some light on the expected trend of the previous expression when increasing the density $\mu$ of end-devices per area, Fig. 2 shows some plots obtained by varying parameter settings. First of all, as summarized in Tab. I, two payload sizes for LoRaWAN packets have been considered, with two corresponding time-on-air values, $\tau_{\min }$ and $\tau_{\max }$ [7], [8]. The plots indicated with circle markers in Fig. 2 correspond to $\tau=\tau_{\min }$, while the other plots are related to the setting $\tau=\tau_{\max }$.

Given a specific setting for $\tau$, the probability $p$ of (1) is easily obtained. Then, $p$ can be substituted in (11): the continuous line refers to the use of a single channel, while the dashed one refers to the use of 3 channels. For the sake of comparison with the throughput expected when a Pure Aloha 
TABLE I

LORAWAN PACKETS.

\begin{tabular}{cc}
\hline Application payload [bytes] & Time-on-air [ms] \\
\hline 1 & $\tau_{\min }=46.336$ \\
222 & $\tau_{\max }=368.896$ \\
\hline
\end{tabular}

access scheme is used without any embedded radiolocation availability, the dotted line plots (6). When using a single channel, the curve of the radiolocation throughput lies under the one related to the throughput of a single-gateway Pure Aloha network: for radiolocation purposes, the probability that a packet is received by at least 3 gateways (thus allowing radiolocation) is lower than the probability that packets are received by at least 1 gateway, as in the classic Pure Aloha scheme. Nonetheless, the availability of 3 channels makes the radiolocation throughput being always higher than that related to a classic Pure Aloha scheme.

Finally, it can be noted that, when $\tau$ increases, the throughput maxima are achieved for lower values of end-device densities.

\section{Simulation RESUlts}

The probability model introduced in the previous section has been validated through extensive simulation results. In order to evaluate the performances of LoRaWAN networks in support to applications for low power radiolocation, realistic traffic patterns have also been used: devices are subject to duty cycle limitations, enforced through an $M / D / 1$ queuing policy implemented on end-devices. In fact, the aim of this section is both to present such results and to discuss the real impact of the LoRa technology for modern low power applications.

To validate the theoretical model through wide simulation campaigns, an ad hoc event-based Python simulator, i.e., LoRaWAN-sim, has been developed in a way to mimic large LoRaWAN deployments, while ensuring processing simplicity. Such an event-based simulator provides an easy way to tune simulation parameters. With the assumption made in the previous section, i.e., the distance between two nearby LoRaWAN gateways is set equal to the transmission range $R$, the resulting probability model does not depend on $R$. Hence, as shown in Fig. 3, the simulated deployment area is large $\sim 10 \times 10 R^{2}$ : gateways are displaced according to an hexagonal lattice (drawn with diamonds), while end-devices are randomly scattered according to a Poisson point process with some intensity $\mu$ (drawn with circles). It has to be noted that the results discussed hereafter are those related to the traffic originated by end-devices falling into the inner rectangle pictured in Fig. 3: this filters out end-devices close to the borders of the simulation area, since the traffic generated by such devices will be less prone to collisions, while the model presented so far assumes an infinite-sized deployment area.

Furthermore, the simulation time $T_{\text {sim }}$ has been fixed to be one hour long for each scenario, and each end-device generates on average a packet every 60 seconds according to

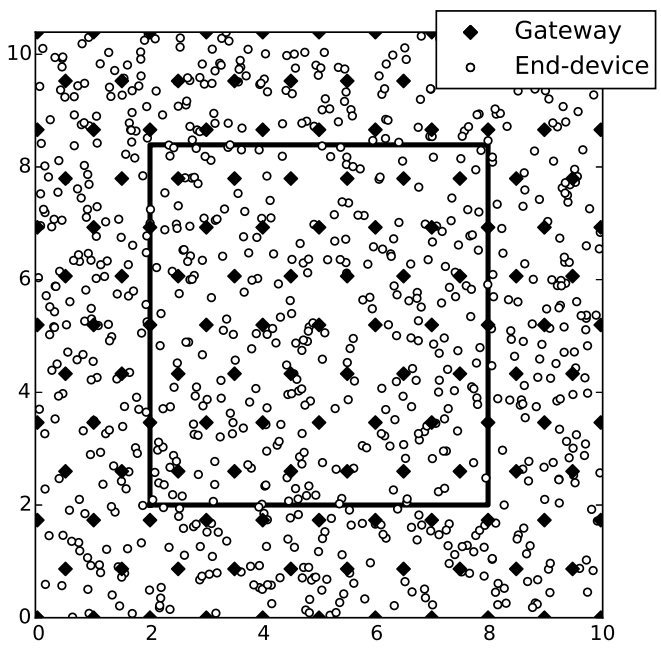

Fig. 3. LoRaWAN deployment example.

an exponential distribution with intensity $\lambda=\frac{1}{60}$. The timeon-air $\tau$ of all packets exchanged during a simulation and the number of available channels $n$ are configured before running any simulation itself.

To picture a general behavior of LoRaWAN networks in handling radiolocation applications, simulations have been run by varying the end-device density $\mu$, the number of channels $n$ and the traffic generation pattern. The latter parameter is varied by considering two values of time-on-air, $\tau_{\min }$ and $\tau_{\max }$.

To achieve statistical significance, 10 different simulation per each parameter setting have been run by feeding the random number generator with 10 different seeds. The results plotted hereafter represent mean values obtained by averaging over the 10 simulation repetitions.

The following subsections summarize and discuss the obtained results in terms of throughput and average time between two radiolocation events.

\section{A. Estimated throughput}

During each simulation, statistics are collected and logged for post-processing purposes. Among them, the number of packets $N^{r}$ sent by any end-device and received by at least 3 gateways during the whole simulation in the area $\mathcal{A}_{\text {sim }}$ bounded by the inner rectangle of Fig. 3 is of utmost importance for radiolocation purposes. Indeed, the portion $\frac{\pi}{\mathcal{A}_{s i m}} N^{r}$ represents the average portion of $N^{r}$ packets sent by any enddevice belonging to a surface extended $\pi$ times an $R$-sided unit square area. Multiplying such a portion with the time-on-air $\tau$, the average amount of time used for useful radiolocation traffic is obtained. By dividing the latter time value by the whole simulation duration, the average occupancy of the radio resource for useful communication is achieved, or, in other words, the estimated throughput $\Delta^{r}$ defined in the following equation:

$$
\Delta^{r}=\frac{\pi \tau}{\mathcal{A}_{\text {sim }} T_{\text {sim }}} N^{r}
$$




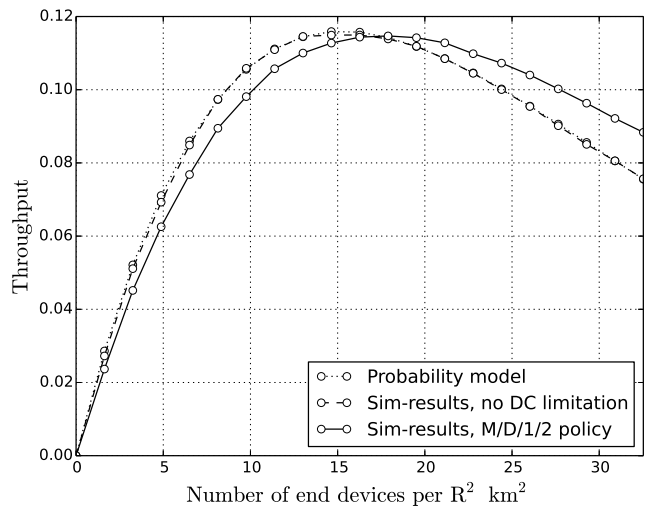

Fig. 4. Throughput of LoRaWAN networks as function of density $(n=1$, $\left.\lambda=1 / 60, \tau=\tau_{\max }\right)$.

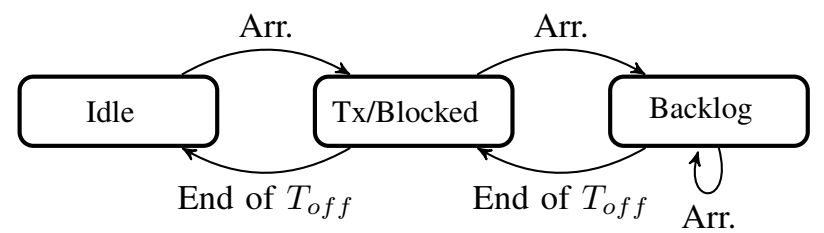

Fig. 5. LoRaWAN transmission model.

Fig. 4 compares the outcome of some simulations with the theoretical model introduced in the previous section. In detail, a single channel has been used $(n=1)$ and the exchanged packets have the maximum possible length, i.e., characterized by a time-on-air equal to $\tau_{\max }$. Substituting the previous values into (11), the dotted line plots the expected throughput $\Gamma^{r}$ for increasing values of the density of devices per unit $R^{2}$ area. At the same time, the dashed line plots the values $\Delta^{r}$ using (12) in which $N^{r}$ was obtained from simulations. The two lines overlap completely, confirming that the probability model introduced in the previous section correctly describes the throughput of low power radiolocation in LoRaWAN networks.

In fact, the LoRaWAN technology is based on the exploitation of the 863-870 MHz ISM band in Europe, which has been policed by ETSI with duty cycle limitations [10]: if the timeon-air of a LoRaWAN packet sent by a given end-device to the closest gateways lasts $\tau$ seconds, then that end-device is required to stay silent for the following $T_{o f f}=(1 / D C-1) \tau$ seconds, where $D C$ is the duty cycle limitation expressed as a number comprised in $[0,1]$. This limitation shapes the traffic generated by an end-device according to the service rate of $M / D / 1$-like queueing systems: the input to the queue is shaped according to an exponential distribution for the interarrival time, with average generation rate equal to $\lambda$ packets per seconds; the service time is deterministic, so that the time between the start of transmission of a packet and that of the following one must at least be $1 / D C$ seconds. In details, it has been assumed that the buffer has sufficient space to backlog a single packet, in other words the system simulated

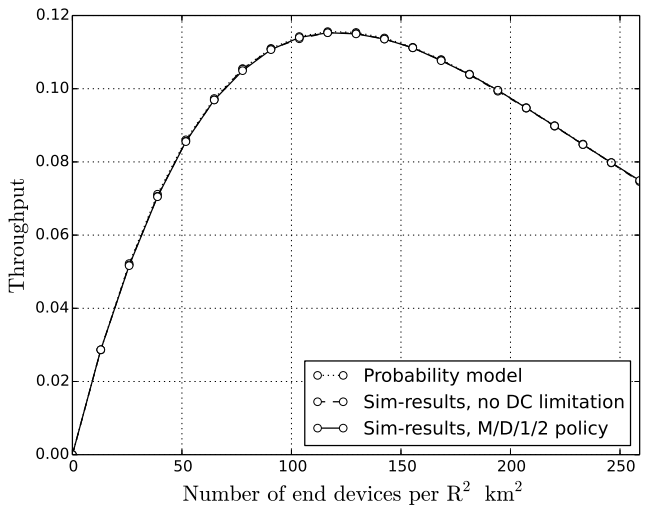

Fig. 6. Throughput of LoRaWAN networks as function of density ( $n=1$, $\left.\lambda=1 / 60, \tau=\tau_{\min }\right)$.

can be labeled as $M / D / 1 / 2$ (as also pictured in Fig. 5). Hence, further simulations have been run by accounting for a duty cycle limitation of $1 \%$, and related results are plot with a continuous line in Fig. 4. From graphical inspection, such line appears as a version of the dashed one (measured throughput when no duty cycle limitation is applied) stretched along the abscissa. Since the maximum throughput is achieved for a given overall traffic offered on the network, such a stretch is reasonably due to a reduced per-device transmission rate. Indeed, end-devices will drop some generated packets from their own buffer according to the $M / D / 1 / 2$ policy: if the end-device is backlogged, i.e., a packet is awaiting to be transmitted at the end of the $T_{\text {off }}$ time, further generated packets during that time period will be dropped.

If the packet time-on-air and the associated $T_{o f f}$ are sufficiently smaller than the average interarrival time associated to packet generation (i.e., $1 / \lambda$ ), all the curves described so far are overlapped, as shown in Fig. 6. In details, the three plots have been obtained for a packet payload size of 1 byte, i.e., for $\tau=\tau_{m i n}$, with a single physical channel used for all communications. Since packets are smaller, it is less likely that packet generation event will happen during the associated $T_{\text {off }}$ time. This translates in a lower probability that packets get backlogged, and even lower and lower probability of dropped packets. In other words, the probability model introduced in the previous section very well approximates the measured throughput in realistic scenarios (i.e., when the duty cycle limitation is enforced) if $\tau \lambda \ll D C$.

It has to be noted that the maximum achievable throughput is around 0.17 in both Figs. 4 and 6 . This value is thus independent from both $\tau$ and $\lambda$. However, for smaller values of $\tau$, the average occupancy of the radio resource is lower, so that many more end-devices can be admitted without compromising the network performances. In more details, when $\tau=\tau_{\max }$ the maximum throughput is achieved for $\mu \sim 15$ end-devices per unit $R^{2}$ area. Interestingly, as verifiable by inspecting Fig. 6, when $\tau=\tau_{\min }$ the maximum throughput is achieved for $\mu \sim 15 \frac{\tau_{\max }}{\tau=\tau_{\min }}=119$ end-devices per unit $R^{2}$ area. 


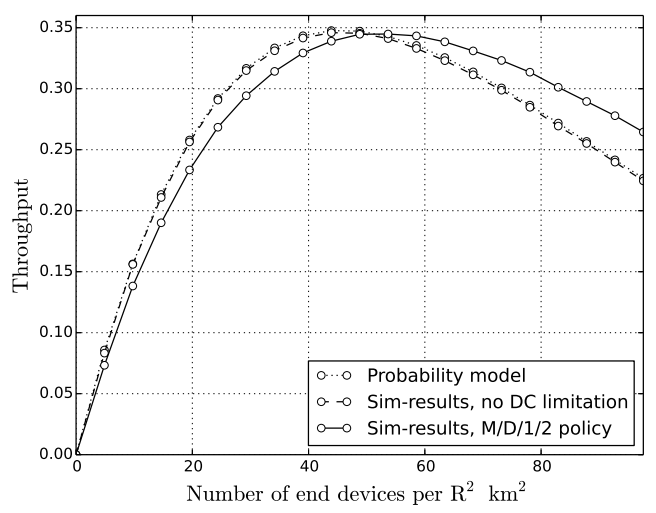

Fig. 7. Throughput of LoRaWAN networks as function of density $(n=3$, $\left.\lambda=1 / 60, \tau=\tau_{\max }\right)$.

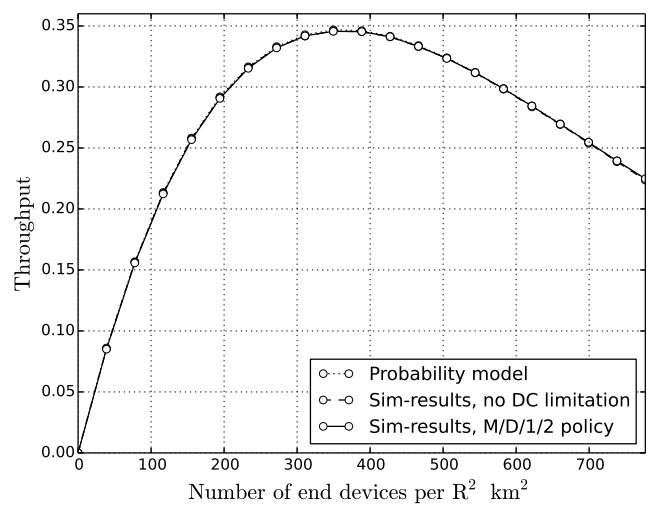

Fig. 8. Throughput of LoRaWAN networks as function of density $(n=3$, $\left.\lambda=1 / 60, \tau=\tau_{\min }\right)$.

Figs. 7 and 8 plot simulation results obtained in similar conditions to respectively Figs. 4 and 6, but with the availability of 3 physical channels. Trends of curves are the same, but the scale of results is different: with 3 channels available, the maximum throughput and the correspondent density of enddevices are 3 time as big as the ones measured in the case of single channel. As matter of fact, the system throughput performances scale with the available number $n$ of channels.

\section{B. Average time between two radiolocation events}

The quality of service of tracking applications relies on the resulting average time between two consecutive radiolocation events. For this reason, it is worth introducing the measure $\bar{T}_{3}$ as the average time between two consecutive events of packet reception by at least 3 gateways; similarly $\bar{T}_{1}$ represents the average time between two consecutive packet receptions by at least 1 gateway. Figs. 9 and 10 show the trend of $\bar{T}_{3}$ and $\bar{T}_{1}$ as function of the density $\mu$ of end-devices, respectively when maximum and minimum packet sizes are used. In both figures, a single channel has been used for communications, with $\lambda=1 / 60$ (i.e., a packet is generated every 60 seconds on average). At a glance, these results show that, increasing

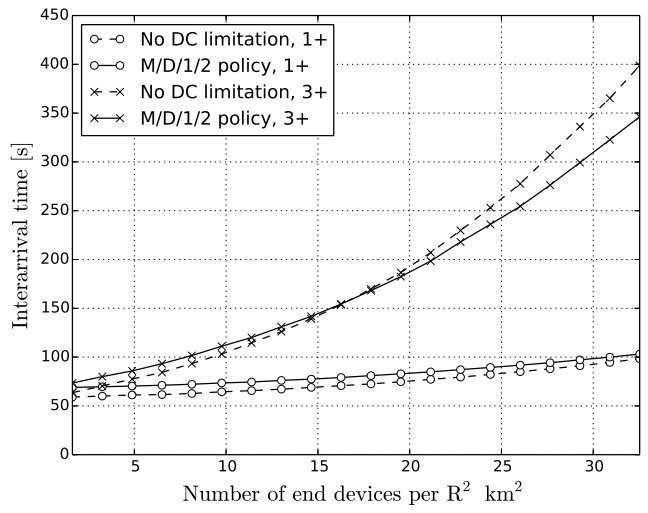

Fig. 9. Time between radiolocation events as function of density ( $n=1$, $\left.\lambda=1 / 60, \tau=\tau_{\max }\right)$.

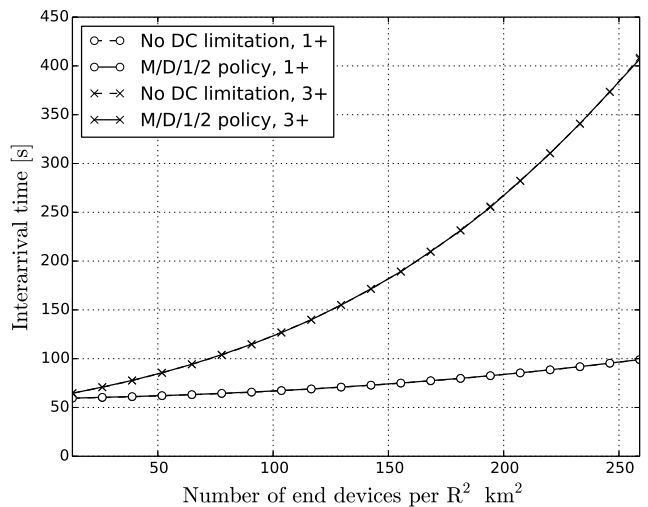

Fig. 10. Time between radiolocation events as function of density ( $n=1$, $\left.\lambda=1 / 60, \tau=\tau_{\min }\right)$.

the density of devices, the considered time value increases according to an exponential law.

Each figure contains four plots: the two ones indicated with 'x' markers are related to $\bar{T}_{3}$, while the other two to $\bar{T}_{1}$; the ones drawn with dashed lines represent simulation results that do not account for any duty cycle limitation, while the other ones are related to simulation accounting for a duty cycle limitation $D C=1 \%$. Clearly, $\bar{T}_{3}$ is greater than $\bar{T}_{1}$, because a packet that is received by at least 3 gateways has been received also by at least 1 gateway, but the opposite is not true. Furthermore, it has to be pointed out that with smaller packets (i.e., $\tau=\tau_{m i n}$, as per Fig. 10) the use of a duty cycle limitation does not make a difference with respect to the lack of such a limitation. However, a difference is definitely visible in Fig. 9 (where $\tau=\tau_{\max }$ ). In details, focusing on the case of $\bar{T}_{3}$, the two related plots cross over for the same density of end-devices that make cross also the corresponding throughput plots of Fig. 4. For densities lower than the crossover point, $\bar{T}_{3}$ is higher when a duty cycle limitation is used, since packet transmissions are more likely backlogged or dropped, thus, delayed. Eventually, Figs. 11 and 12 report simulation results 


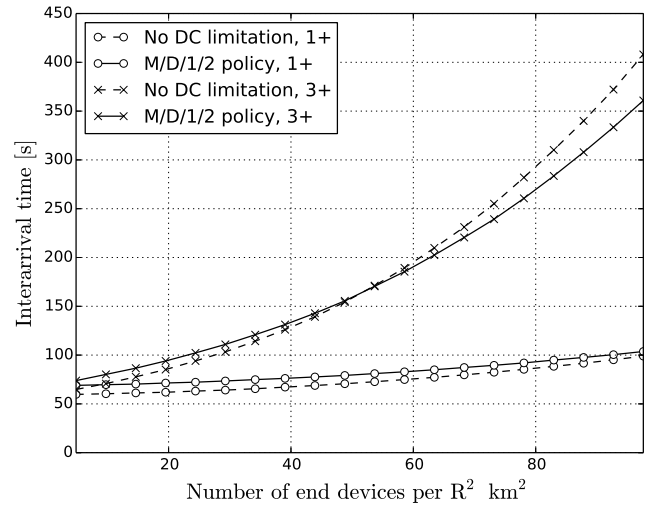

Fig. 11. Time between radiolocation events as function of density ( $n=3$, $\left.\lambda=1 / 60, \tau=\tau_{\max }\right)$.

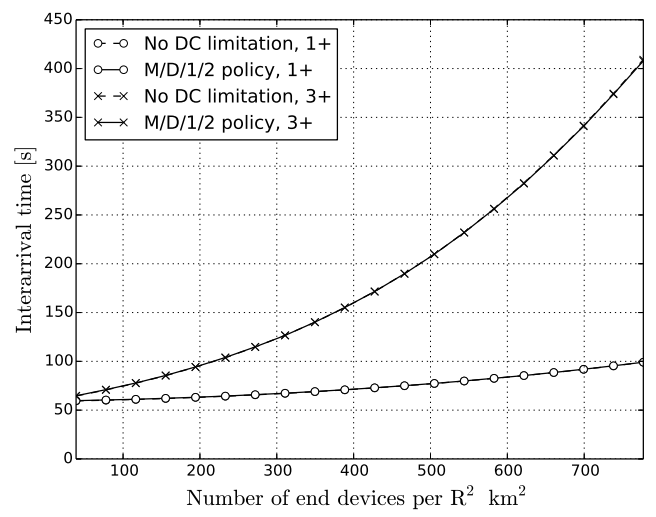

Fig. 12. Time between radiolocation events as function of density ( $n=3$, $\left.\lambda=1 / 60, \tau=\tau_{\text {min }}\right)$.

related to $\bar{T}_{3}$ and $\bar{T}_{1}$ in the case of use of 3 channels.

From the analysis of all the plots related to the average time between two consecutive radiolocation events when no duty cycle limitation is applied, it is evident that $\bar{T}_{3}$ is $\sim 145$ seconds in correspondence of the end-device density that maximizes the throughput. Indeed, $\bar{T}_{3}$ is proportional to the interarrival time between packet generations, $1 / \lambda$, that has been fixed for all simulations to 60 seconds. As a matter of fact, when a duty cycle limitation is applied and $\tau=\tau_{\max }$, some generated packets are backlogged or discarded, so that the effective interarrival time between packets transmitted on the radio is higher. As a consequence (see Figs. 9 and 11), the value of $\bar{T}_{3}$ in correspondence of the end-device density that maximizes the throughput is also higher (i.e., $\sim 150$ seconds).

\section{CONCLUDING REMARKS AND FUTURE WORKS}

In this paper, a probabilistic model for radiolocation in LoRaWAN networks has been proposed. In this model, nodes are scattered randomly on a plane and base stations are placed in a deterministic way on intersection points of a tessellation performed with equilateral triangles. Nodes send radiolocation packets on a channel using the Aloha access scheme, and for a packet to be successful, it has to be received by at least three gateways. For a simplified version of this model without dutycycle limitation, a closed form expression for the probability of success was derived. Extensive simulations were performed taking into account a more realistic assumption of duty cycle limitation. The results derived from the analysis match well the simulation results when the duty cycle is close to zero.

The ongoing work focuses on obtaining theoretical results for the model with duty cycle limitation. This model will involve investigation of a network of $M / D / 1 / K$ queues. For the future work, more advanced features of the network should be taken into account. These include mobile nodes and radio interference from other technologies. These results will be validated in real scenarios.

\section{ACKNOWLEDGMENTS}

This work has been co-funded by a FEDER-FSE 2014-2020 fund of the Région Midi-Pyrénées and the European Union and also by French Government (program: investment for future) in the project: Smart Services for Connected vehiCles - S2C2.

\section{REFERENCES}

[1] B. Warneke, M. Last, B. Liebowitz, and K. S. J. Pister, "Smart Dust: communicating with a cubic-millimeter computer," IEEE Computer, vol. 34, no. 1, pp. 44-51, Jan. 2001.

[2] "IEEE Standard for Low-Rate Wireless Networks," IEEE Std 802.15.42015 (Revision of IEEE Std 802.15.4-2011), Apr. 2016.

[3] M. R. Palattella, N. Accettura, X. Vilajosana, T. Watteyne, L. A. Grieco, G. Boggia, and M. Dohler, "Standardized Protocol Stack for the Internet of (Important) Things," IEEE Commun. Surveys Tuts., vol. 15, no. 3, pp. 1389-1406, 2012.

[4] R. Sanchez-Iborra and M.-D. Cano, "State of the Art in LP-WAN Solutions for Industrial IoT Services," Sensors, vol. 16, 2016.

[5] IETF Working Group, "IPv6 over Low Power Wide-Area Networks (lpwan)," 2016. [Online]. Available: http://tools.ietf.org/wg/lpwan/

[6] M. R. Palattella, M. Dohler, L. A. Grieco, G. Rizzo, J. Torsner, T. Engel, and L. Ladid, "Internet of Things in the 5G Era: Enablers, Architecture, and Business Models," IEEE J. Sel. Areas Commun., vol. 34, no. 3, pp. 510-527, Mar. 2016.

[7] LoRa Alliance, "LoRaWAN Specification v1.0.2," Jul. 2016.

[8] "SXI272/3/6/7/8 LoRa Modem Design Guide AN1200.13 Revision 1," Jul. 2013.

[9] J. Petäjäjärvi, K. Mikhaylov, A. Roivainen, T. Hanninen, and M. Pettissalo, "On the coverage of LPWANs: range evaluation and channel attenuation model for LoRa technology," in ITS Telecommunications (ITST), 2015 14th International Conference on, Dec. 2015, pp. 55-59.

[10] "ETSI EN 300 220-2 V2.4.1," European Telecommunications Standards Institute (ETSI), May 2015.

[11] N. Abramson, "THE ALOHA SYSTEM: another alternative for computer communications," in Proc. of the fall joint computer conference. ACM, 1970, pp. 281-285.

[12] L. Vangelista, A. Zanella, and M. Zorzi, Long-Range IoT Technologies: The Dawn of LoRa ${ }^{T M}$. Springer International Publishing, 2015, pp. 51-58.

[13] K. Mikhaylov, J. Petäjäjärvi, and T. Haenninen, "Analysis of Capacity and Scalability of the LoRa Low Power Wide Area Network Technology," in European Wireless, May 2016, pp. 1-6.

[14] F. Adelantado, X. Vilajosana, P. Tuset-Peiró, B. Martínez, and J. Melià, "Understanding the limits of LoRaWAN," CoRR, vol. abs/1607.08011, 2016.

[15] LoRa Alliance, "LoRaWAN Regional Parameters v1.0," Jul. 2016.

[16] D. Bajović, D. Jakovetić, D. Vukobratović, and V. Crnojević, "Slotted Aloha for networked base stations," in Proc. IEEE Int. Conf. on Communications Workshops (ICC 2014), Jun. 2014, pp. 520-526.

[17] L. G. Roberts, "ALOHA Packet System with and Without Slots and Capture," SIGCOMM Comput. Commun. Rev., vol. 5, no. 2, pp. 28-42, Apr. 1975. 